\section{Ophthalmopathic immunoglobulin not detected in patients with Graves' ophthalmopathy}

SIR,-Endocrine ophthalmopathy is sometimes a serious condition and may present several problems of management. Efforts have been made to develop assays to monitor the activity of the disease. There is no simple and useful test available today. Recently Kendall-Taylor et al reported on specific antibodies present in sera of patients with active eye disease.' These antibodies reacted with preparations of solubilised porcine eye muscle or guinea pig harderian gland in an enzyme linked immunosorbent assay. Subsequently, anothe group described, also in this journal, some confirmatory data based on a similar technique.

These promising reports prompted us to estab lish an analogous assay for ophthalmic immunoglobulin. However, we have not been able to achieve any positive data in support of these previous reports and would like to report on our experiences here.

W'e have used exactly the methods detailed by Kendall-Taylor et al ${ }^{1}$ emploving porcine eve muscle antigenic material obtained fresh at slaughter which was homogenised on ice in separate experiments with the Ultra-Turrax as well as glass-Teflon procedures. We then tested sera of patients with very active endocrine ophthalmopathy $(n=10$ in enzyme linked immunosorbent assay. These patients all had high titres of thyroid stimulating hormone recepto antibodies as determined by the TRAK-assay 6 (52) U/l, mean (SD); Henning Berlin Ltd, Berlin, West Germany). We also examined sera of healthy individuals $(n=4)$ as well as sera of patients with othe continuing autoimmune disorders such as systemic lupus erythematosus $(n=5)$, primary biliary cirrhosi $(n=5)$, chronic active hepatitis $(n=5)$, rheumatoid arthritis $(n=4)$, and thyroiditis ( $n=4$; sera with high titres of microsomal and thyroglobulin antibodies; Wellcome Ltd haemagglutination test)

Most of the serum samples from patients with primary biliary cirrhosis gave absorbances on assa higher than those of other patients $\left(\mathrm{E}_{405}=1 \cdot 15\right.$ $(0.627) v 0.345(0.113))$ or healthy individuals $\left(\mathrm{E}_{+05}=\right.$ $0.302(0.027))$.

Probably these results were due to antibodies binding to mitochondrial antigens in the $105000 \mathrm{~g}$ pellet used for coating the microtitre wells. Such "false positive" binding with sera from patients with primary biliary cirrhosis was observed also when $105000 \mathrm{~g}$ pellet of skeletal muscle was used and to a smaller extent with corresponding material from liver homogenates. Subsequently, we modified the methods and tested also porcine eye muscle materia sedimenting at $10000 \mathrm{~g}$, as well as the effect of solubilisation of the $105000 \mathrm{~g}$ pellet with a detergen $(1 \%$ Triton X-100) before coating. Also in some sets of experiments we included $0.5 \%$ Tween 20 in the buffers used in the washing and dilution steps. Finally, we absorbed homogenised eve muscle material on a lectin column (Lentil-Sepharose, Pharmacia) and coated microtitre plates with material

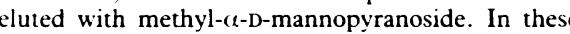
experiments, no specific antibody binding could be shown with sera of patients with active endocrine ophthalmopathy exceeding that of sera from healthy controls.

Thus, we have been unable to reproduce the results of Kendall-Taylor et al using either the identical or modified assays. The reason for this discrepancy is at present unclear. The role of specific antibodies in the pathogenesis of endocrine ophthalmopathy remains obscure and needs to be substantiated.

KRISTINA BJELKENKRANTZ ANDERS KARLSSON

Ib MENDEL-HARTVIG THOMAS H TÖTTERMAN

Department of Internal Medicine and

Secrion of Clinical Immunology,

University Hospital, and Department of Biochemistry and

Immunology,

Pharmacia, Uppsala, Sweden
1 Kendall-Taylor $\mathrm{P}$, Atkinson S, Holcombe $\mathrm{M}$. A specific $\mathrm{IgG}$ in Graves' ophthalmopathy and its relation to retro-orbital and thyroid autoimmunity. Br Med T 1984;288:1183-6.

2 Faryna M, Nauman J, Gardas A. Measurement of autoantitundies against human eve muscle plasma membranes in Graves optrthalmopathy. Br Med J 1985;290: 191-2.

\section{Code of practice for the Mental Health} Act 1983

SIR,-The leading articles by Dr John R Hamilton and Dr Denis Pereira Gray (10 May, p 1219) are timely in warning of the potential restrictions to the practice of medicine if the widely drawn draft code of practice by the Mental Health Act Commission is not greatly modified

The BMA mental health group considers that the commission is wrong to give expressions of opinion which go beyond the Act-for example, that social workers should be concerned in every case of compulsory hospital admission even when the applicant is a "nearest relative." In addition, the contents of such a detailed "code of practice," amounting to 209 pages, may become enshrined as common law and force doctors to practise defensive medicine by complying with each sentence of the code.

If accepted by the Department of Health and Social Security the code will apply to all mentally ill people, including the $80 \%$ in the community who are being looked after by their general practitioners. It is interesting that the latter are mentioned only once in the code.

It can well be imagined that if allowed to go unchallenged such codes of practice may be drawn up for all aspects of medical practice. It is therefore the direct concern of the whole profession. The British Medical Association is to be congratulated on deciding to seek legal advice on the remit of the commission under section 118 of the Act.

ARNOLd ElliotT

Ilford,

Essex IG2 7LE

\section{Occupational asthma due to methyl methacrylate bone cement}

SIR, - We read with great interest the recent repor of Dr C A C Pickering and colleagues (24 May, p 1362), but the report could have been used to highlight the many other problems associated with methyl methacrylate cement.

Orthopaedic surgery is currently plagued with an increasing number of revision cemented hip replacements, resulting in less available operating time for the much needed primary procedure. This is due to a number of problems, including poor prosthesis design, inadequate technique, and in correct patient selection

However, the mechanical performance of methyl methacrylate bone cement is vastly different from that of bone and can cause considerable problems at the cement-bone interface, with gradual bone resorption and eventual loosening. This may be related to its ability to attract macrophages. ${ }^{.}$Thermal necrosis is also a problem, though only when larger cement masses are used, as in tumour replacement or revision surgery. ${ }^{2}$ It has certainly been shown to burn the patient's skin if left carelessly on top of the drapes while curing.

From the allergic viewpoint, contact dermatitis appeared among dentists in the early 1950s and later among orthopaedic staff, ${ }^{4}$ methyl methacrylate being a strong lipid solvent and penetrating rubber gloves very rapidly.

Reports in the early 1970s were full of the cardiorespiratory side effects of orthopaedic bone cement, which was implicated as a cause of fat embolism $^{6}$ and cardiac arrest. This has generally been held to be due to monomer diffusing out of the polymerising cement mass and is a particular problem if preoperative fluid volume is not controlled. Such monomer may also result in a "cement hepatitis"' manifest as raised $\gamma$-glutamyltransferase activities in patients after hip replacement.

Recognition of the toxic effects of monomer inhalation is not new. Toxicity, including embryooxicity, has been recognised in laboratory animals. ${ }^{8}$ It is alarming to think that a dental technician can inhale the vapour for several hours at a time in a confined area. Fortunately, no directly carcinogenic effects can be attributed to the material, though sarcomatous degeneration has been seen in animals ${ }^{9}$ and one case of chondrosarcoma has appeared in man. ${ }^{10}$

Given the above, it is truly wonderful that methyl methacrylate has lasted as long as it has. For ourselves, we shall continue to use it until something better comes along as we know that, given meticulous technique, aseptic loosening rates of $2 \%$ at 10 years are not unreasonable.

R N VILLAR F G JOHNSTON P M SCOTT

Deparement of Orthopaedic Surgery,

Addenbrooke's Hospital

Cambridge CB2 2QQ

I Freeman MAR, Bradley GW', Revell PA. Observations upon the interface between bone and polymethylmethacrylate cement. I Bome foint Surg [Br] 1982;64:489-93.

2 Mjoberg B, Pettersson H, Rosenqvist R, Rydholm A. Bone cement, thermal injury and the radiolucent zone. Acta Orthop Scand 1984:55:597-600.

3 Jefferiss CD Avoidable complication of McKee-Farrar arthroplasty. BrMed J 1971;ii:382.

Fisher AA. Acrylic bone cement sensizaion and dermatisis. Cutis 1973;12:333-7.

Waegemaekers THJM, Seutter E, Den-Arend JACJ, et al. Permeability of surgeons' gloves to methyl methacrylate. Acl Orthop Scand 1983;54:790-5.

6 Dandy DJ. Fat embolism following prosthetic replacement of the femoral head. Injuny 1971;3:85-8.

Ritter MA, Gioe TJ, Sieber JM. Systemic effects of polymethy methacrylate. Acta Orthop Scand 1984;55:411-3.

8 Nicholas CA, Lawrence WH, Autian J. Embryotoxicity and fetotoxicity from maternal inhalation of methyl methacrylate monomer in rats. Toxicol Appl Pharmacol 1979;50:451-8.

9 Contzen H, Straumann F, Paschke E. Grundlagen der Alloplastik mit Metallen und Kunststoffen. Stuttgart: G Thieme Verlag, 1967.

10 Thompson JR, Entin SD. Primary extraskeletal chondrosar coma. Report of a case arising in conjunction with extrapleural lucite ball plombage. Cancer 1969;23:936-9.

\section{Antibody response and clinical reactions in} children given measles vaccine with immunoglobulin

SIR,-I was interested and pleased to read the paper by Dr S Lingam and colleagues (19 April, p 1044) describing how a number of children with neurological disorders were given measles immunisation and measles immunoglobulin with no untoward reactions. Anything that can be done to increase the immunisation rates for diseases such as measles and whooping cough is to be supported. This is especially so for children who are thought to be at increased risk of complications from these diseases because of pre-existing neurological abnormalities.

While showing that children given simultaneous mmunoglobulin with their measles immunisation seemed to have few adverse reactions, this does not in any way prove that the immunoglobulin has had any useful effect. The current attenuated vaccines in use are further attenuated than the Edmonston $B$ vaccine and cause fewer reactions. It may be that the immunoglobulin is not necessary, and I think this would be a suitable topic for further study.

Antibody levels were measured in their patients 